\title{
Adaptation and constraint in the evolution of the mammalian backbone
}

\author{
Katrina E. Jones ${ }^{1 *}$ (D), Lorena Benitez ${ }^{1}$, Kenneth D. Angielczyk ${ }^{2}$ and Stephanie E. Pierce ${ }^{1 *}$
}

\begin{abstract}
Background: The axial skeleton consists of repeating units (vertebrae) that are integrated through their development and evolution. Unlike most tetrapods, vertebrae in the mammalian trunk are subdivided into distinct thoracic and lumbar modules, resulting in a system that is constrained in terms of count but highly variable in morphology. This study asks how thoracolumbar regionalization has impacted adaptation and evolvability across mammals. Using geometric morphometrics, we examine evolutionary patterns in five vertebral positions from diverse mammal species encompassing a broad range of locomotor ecologies. We quantitatively compare the effects of phylogenetic and allometric constraints, and ecological adaptation between regions, and examine their impact on evolvability (disparity and evolutionary rate) of serially-homologous vertebrae.

Results: Although phylogenetic signal and allometry are evident throughout the trunk, the effect of locomotor ecology is partitioned between vertebral positions. Lumbar vertebral shape correlates most strongly with ecology, differentiating taxa based on their use of asymmetric gaits. Similarly, disparity and evolutionary rates are also elevated posteriorly, indicating a link between the lumbar region, locomotor adaptation, and evolvability.

Conclusion: Vertebral regionalization in mammals has facilitated rapid evolution of the posterior trunk in response to selection for locomotion and static body support.
\end{abstract}

Keywords: Vertebral column, Geometric morphometrics, Mammal evolution, Ecology, Locomotion, Adaptation

\section{Background}

Disentangling the processes that lead to the evolution of complex biological structures is one of the major aims of evolutionary biology [1-4]. Integration between separate component parts of anatomical structures has been increasingly recognized as an important determinant of variation on genetic, phenotypic, and evolutionary scales [5-11]. Patterns of integration may enhance evolvability by compartmentalizing functional units into modules, limiting pleiotropic gene effects and thus promoting independent evolution in response to divergent selective regimes [4, 12-17]. The vertebrate axial skeleton represents the archetypal integrated structure because it is composed of repeating, serially-homologous vertebrae that have maintained remarkable self-similarity throughout vertebrate history $[18,19]$. Simultaneously, the subdivision

\footnotetext{
* Correspondence: katrinajones@fas.harvard.edu; spierce@oeb.harvard.edu ${ }^{1}$ Museum of Comparative Zoology and Department of Organismic and Evolutionary Biology, Harvard University, 26 Oxford Street, Cambridge, MA 02138, USA

Full list of author information is available at the end of the article
}

of the vertebral column into semi-autonomous modules (regions) has been achieved to varying degrees across different groups, with mammals constituting the best-known and most-extreme example [20-24]. Thus, the vertebrate axial skeleton provides a system with which to examine the interaction between integration, modularity, and morphological evolution over macroevolutionary timescales.

Evolution of the vertebral column is shaped not only by integration between vertebrae (serial homology), but also by phylogenetic and allometric constraints, as well as adaptation to diverse ecological niches. Phylogenetic history is a key predictor of vertebral variation, reflecting shared genetic, developmental, or functional interactions [25-28]. Allometry is also an important factor, as structural adaptation of vertebrae to static loading is necessary to accommodate increasing size [25, 27, 29-32]. The axial skeleton is integrally involved in body support and movement, respiration, and anchoring the head and limbs and, therefore, is subject to selection for a variety of functions and ecological specializations [22, 28, 33-35]. Further, evolutionary responses to these factors may be 
shaped by the modular organization of the vertebral column, resulting in serial modifications of morphological variation and disparity $[24,26,30,36]$.

Here we examine evolution of the mammalian 'trunk' (thoracic and lumbar vertebrae) to understand how regionalization of the vertebral column impacts macroevolutionary patterns. In most tetrapods, the vertebral column is relatively homogenous and vertebral counts vary widely between species. In mammals, however, the vertebral column is constrained in vertebral count but extremely variable in terms of vertebral morphology resulting in discrete regions [37-40]. Most notably, the functional subdivision of the mammal trunk (thoracolumbar region) into respiratory (thoracic) and locomotor (lumbar) regions in the synapsid forerunners of mammals is a critical step in establishing the mammalian bauplan; demarcating the origin of novel vertebral functions and locomotor behaviors [20,39, 41-44]. Whereas the thoracic region forms part of the ribcage, facilitating breathing and supporting the forelimb, the novel mammalian lumbar region is free of ribs and has been linked with various locomotor behaviors (e.g., hunting behavior [45], arboreality [46], posture [47, 48], running performance [49], jumping [50], swimming [33]). Therefore, the mammal trunk provides the ideal case study for understanding the implications of regionalization on vertebral variation, adaptation, and evolvability.

Our study asks how has thoracolumbar regionalization impacted adaptation and evolvability through mammalian evolution? To answer this question, we measured vertebral morphology in a wide range of extant mammals, spanning a variety of clades and sizes. We quantified the shape of five thoracolumbar vertebrae, selected to reflect variation among and between regions, using 3D geometric morphometrics and compared the influence of phylogeny, allometry, and ecology, as well as modularity, disparity and evolutionary rates along the column. Based on prior work, we predicted that phylogenetic history, allometry, and/or ecology would have significant effects on the overall morphology of the thoracolumbar region (Hypothesis 1), but that ecology would have greater influence in the lumbar region (Hypothesis 2). We also predicted that morphological variation would reflect the modular organization of the thoracolumbar region (Hypothesis 3), and if modularity and adaptation promote evolvability, that the lumbar region would display elevated disparity and evolutionary rates (Hypothesis 4).

\section{Methods}

\section{Specimens and sample size}

Vertebrae from 52 mammalian species, including monotremes, metatherians, and representatives from all major eutherian clades, were selected to span mammalian phylogeny and to represent a broad diversity of locomotor ecologies (Fig. 1). Although some groups are relatively more abundant in collections (e.g., ungulates, carnivores), we attempted to sample evenly across the mammalian tree to avoid bias toward groups. Our sample size and selection of representatives from each major clade are comparable to a recent study that examined morphological divergence and evolvability in ray-finned fishes using similar methods [17]. A phylogeny for the mammalian taxa sampled was generated using timetree.org [51], which utilizes a hierarchical synthesis of published molecular timetrees to estimate topology and branch lengths (Fig. 1).

Specimens examined were primarily from the osteological collections of the Museum of Comparative Zoology (MCZ), Harvard University (Additional file 1: Table S5). Digital images of several specimens were also

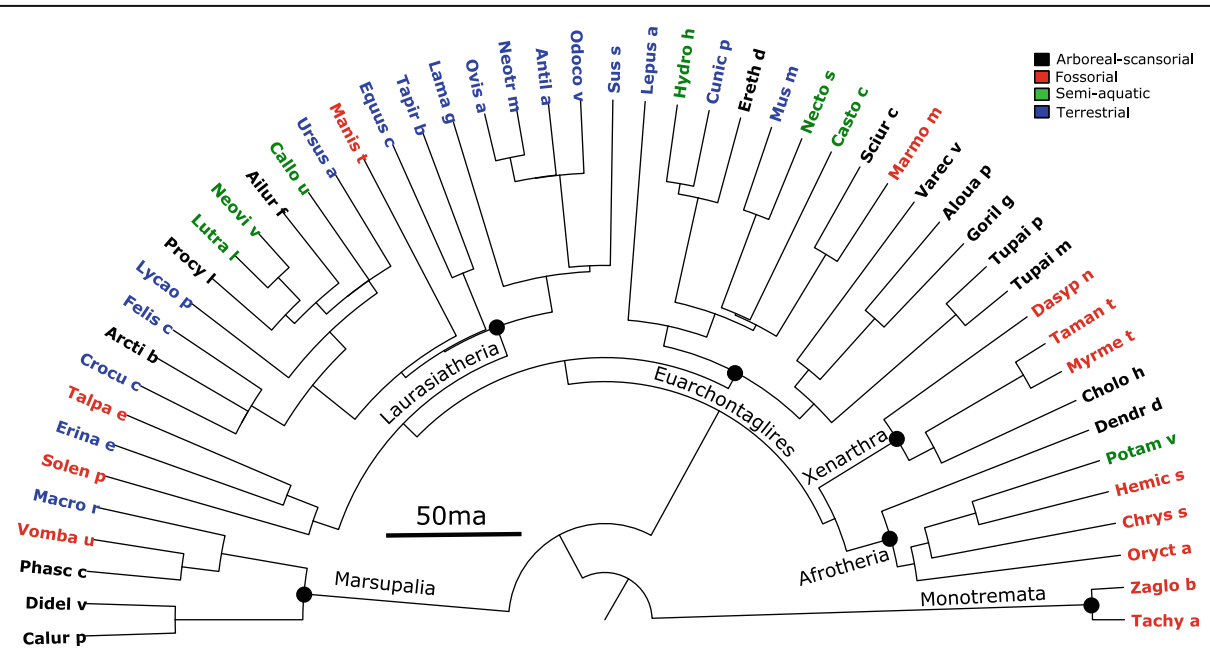

Fig. 1 Time-calibrated phylogeny of the species included in this study. Based on a synthesis of relationships and branch lengths from a wide range of molecular studies [51]. Branch lengths scaled to time in millions of years. For full taxonomic names see Additional file 1: Table S6 
obtained from the online repositories 'Morphosource' and 'Digimorph', originally collected from the American $\mathrm{Mu}$ seum of Natural History (AMNH) and the Field Museum of Natural History (FMNH) (Additional file 1: Table S5). All specimens analyzed were adults (based on fusion of epiphyses), pathology-free, and with vertebrae accurately seriated (i.e. precise identification of the anterior-posterior position along the column).

This study utilized a broad phylogenetic sample to examine large-scale patterns across mammals. Therefore, we used very broad groupings to tease apart large-scale influences of ecology and applied multiple ecological assignments where appropriate. Information on locomotor ecology was gathered from the literature (see Additional file 1: Table S1 for literature sources), and species were classified into four categories according to the definitions used in previous studies [52]: terrestrial, scansorial-arboreal, fossorial and semi-aquatic. We combined scansorial and arboreal into a single group of climbing-specialized mammals to increase within-group sampling. Flying (e.g. bats) and fully aquatic (e.g. whales) taxa were not included due to their derived locomotor habits and the highly disparate selective pressures they likely encounter. A secondary locomotor classification was also defined for taxa which utilize multiple locomotor strategies based on behavioral descriptions or had multiple classifications in the literature (Additional file 1: Table S5).

To capture maximum shape variation along the vertebral column and compare taxa with varying vertebral counts, five thoracolumbar vertebrae per specimen were sampled, resulting in a total of 260 measured vertebrae. The vertebrae selected included: the first thoracic, the numerically mid-thoracic, the vertebra that marks the transition from horizontally- to vertically-oriented zygapophyses (diaphragmatic), the vertebra at one-third of lumbar length (anterior lumbar), and the final lumbar vertebra. As morphology varies strongly between vertebral regions, vertebrae were defined relative to regional landmarks (e.g., first free rib, diaphragmatic, last free rib) to enable identification of functionally homologous positions in taxa with varying vertebral formulas (Additional file 1: Figure S1). For a full description of the vertebrae selected for each species see Additional file 1: Supplemental Materials and Methods.

\section{Data acquisition and landmarks}

A three-dimensional geometric morphometric (GMM) approach was used to quantify the complex shape of the thoracolumbar vertebrae. For structures present throughout the column (centrum, arch, zygapophyses, neural spine) traditional homology-based landmarks were selected to capture shape variation (Fig. 2, Additional file 1: Table S1). The curvature of the endplate lacks suitable features for landmarking but is an important feature in determining

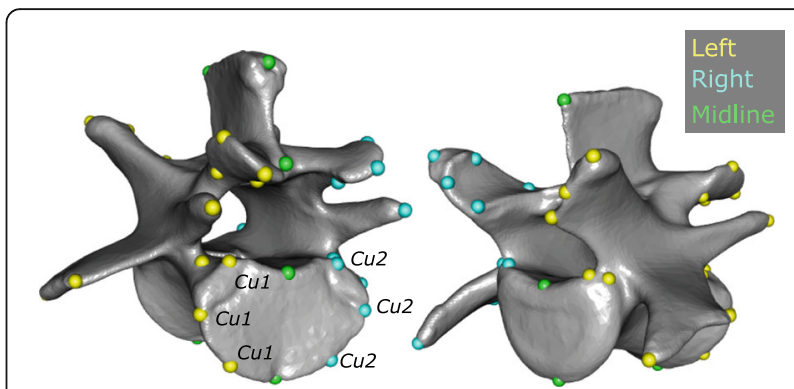

Fig. 2 Three-dimensional landmarks collected on second lumbar vertebra of Marmota monax. For descriptions see Tables S1 and S2. cu: curve

articulation of the intervertebral joints. Therefore, sliding semi-landmarks were employed to capture the shape of the endplate [53]. Further, some muscular processes (anapophysis, metapophysis, transverse process) are variably present along the column, but represent a vital component of vertebral morphology. To include these structures, we adopted a redundant landmarking approach (following e.g. $[54,55])$, which uses overlapping landmarks to record the presence or absence of serially-homologous structures along the column (Additional file 1: Table S2). For further details see Additional file 1: Supplementary Materials and Methods.

Large-bodied specimens (e.g., Equus caballus) were landmarked using a MicroScribe G2X Digitizer. For smaller specimens, landmarks were collected from three-dimensional models created using a Skyscan model 1173 micro-computed tomography (CT) scanner in the MCZ or from online repositories (see above). The target vertebrae were manually segmented and 3D rendered using Mimics software (Materialise, version 19) to make 3D surface meshes. Thirty-eight landmarks were placed manually using the MicroScribe or the 'Measure and Analyze' feature of the Simulate menu of Mimics. For the sliding semi-landmarks, two curves with greater than 10 landmarks each were collected along the left and right sides of the caudal endplate. The curves were later resampled to five landmarks each using the function 'digit.curves' in geomorph [56, 57]. The first and last landmarks of each curve were then replaced by single fixed dorsal and ventral midline centrum landmarks (Additional file 1: Table S1), ensuring symmetry when the semi-landmarks were slid between them. Thus, a total of $443 \mathrm{D}$ coordinates were collected for each vertebra (38 fixed landmarks and two curves with three sliding semi-landmarks each) (Fig. 2; see Additional file 1: Tables S1 and S2 for landmark descriptions). An error study was conducted on four species with four replicates, which demonstrated that landmarking accuracy was sufficient to distinguish both species and vertebrae using this protocol (see Additional file 1: Supplementary Material and Methods, Figure S2, Table S3). 


\section{Data analysis}

All analyses were conducted in $\mathrm{R}$ version 3.4.2 and the package geomorph version 3.0.5 [56, 57]. Landmark coordinates were aligned using generalized Procrustes superimposition (GPA) with the function 'gpagen' to remove size, rotation, and translation, and the semi-landmarks were permitted to slide to maximize bending energy. To remove asymmetry from the dataset, symmetrized Procrustes coordinates were generated using 'bilat.symmetry'. Analyses were conducted on each of the five individual vertebral positions, separately, and on a 'whole-column' dataset (all vertebral positions combined per specimen) as described below.

\section{Individual vertebral positions Shape variation}

GPA and Principal components analysis (PCA) were used to visualize vertebral shape variation at the different vertebral positions - separately - using 'plotTangentSpace'. Shape variation at each vertebral position was visualized using mesh warping, based on the 3D surface mesh of the specimen lying closest to the mean shape for that vertebral position. Meshes were morphed to the mean shape for each position using 'mshape' and 'warpRefMesh' and then to PCA extremes or ecological grouping means using 'plotRefToTarget'.

\section{Phylogeny, size and ecology}

The multivariate K-statistic was used to estimate phylogenetic signal present in the symmetrized Procrustes coordinates [58, 59]. This was calculated using the function 'physignal' with 10,000 permutations to determine significance. Higher K-values correspond to a stronger phylogenetic signal, with values greater than one indicating that traits are conserved within the phylogeny, whereas values less than one indicate weaker phylogenetic signal and more convergence. To quantify the relationship between vertebral shape, size, and ecology, we used phylogenetically-corrected multivariate analysis of covariance (MANCOVA) in 'procD.pgls' [60, 61]. In this model, symmetrized Procrustes coordinates were the dependent variables with log centroid size (as a proxy for body size) as a covariate and ecological grouping (Additional file 1: Table S5) as a factor. As some taxa exhibit behaviors consistent with multiple ecological groups, the MANCOVA was run for both the primary and secondary ecological grouping assignments.

\section{Modularity}

Phylogenetically-corrected modularity was calculated as the CR coefficient, a ratio of the between-vertebra covariation to the within-vertebra covariation that reflects the relative independence of the vertebrae, using 'phylo.modularity' [62]. Pairwise variations in CR coefficient along the column were visualized using 'corrplot'. To ensure the dataset was appropriate for this analysis, stability of the covariance matrix for each vertebra was confirmed using random skewers analysis with 1000 bootstrap replicates (median $r=0.95$ ) [63].

\section{Disparity and evolutionary rate}

Morphological disparity was quantified for each vertebral position using the 'morphol.disparity' function. Procrustes variances for each position were calculated, with log centroid size included as a covariate, and differences between vertebrae were tested using permutation [53]. Further, evolutionary rates at each vertebral position were calculated using 'compare.multi.evol.rates' [64], and the significance of between-position differences tested using phylogenetic simulation. Confidence intervals on disparity and rate measures were calculated based on randomized residuals from a linear model using 'procD.lm' and 'procD.pgls'.

\section{Whole-column analysis Shape, phylogeny, and ecology}

The vertebral column, though composed of individual vertebrae, functions as a unit and is developmentally and evolutionarily integrated [22]. Therefore, in addition to examining the evolution of individual vertebrae, it is also important to consider evolutionary influences on the whole thoracolumbar column. To examine the evolution of multiple vertebrae simultaneously, we conducted a combined analysis of all five vertebral positions. Procrustes-aligned landmarks (single fit for all vertebrae) from each of the five vertebral positions for each species were concatenated to produce a new 'whole-column' coordinate set. To reduce the dimensionality of the dataset, only midline and left-side landmarks were used, resulting in a total of 130 landmarks for each specimen, which is within the dimensionality range of other comparative morphometric studies [65-67]. This 'whole-column' landmark set was then subjected to the shape, phylogenetic signal, and MANCOVA analyses described above to examine the influence of phylogeny, size, and ecology on total thoracolumbar evolution. The residual randomization tests employed by 'ProcD.pgls' are specifically designed for, and are robust to, high dimensionality data $[60,68]$. As each vertebra was treated separately in the initial Procrustes superimposition, the relative contribution of each vertebral position to the whole-column was examined by summing the absolute PC loadings of each landmark across the individual vertebral position. Whole-column PC axes reflect shape variation in all five vertebral positions simultaneously. Therefore, shape variation along $\mathrm{PC}$ axes was visualized by extracting loadings for each individual vertebral positions from the concatenated PC loadings, and warping the vertebrae as described above. 


\section{Method validation}

Previous studies quantifying the shape of multiple elements simultaneously have either combined PC or relative warps scores from separate analyses to produce new shape variables $[27,69,70]$. Due to the common landmarking approach for each vertebra taken here, we were able concatenate Procrustes-coordinates directly, producing a mathematically-identical shape space to that obtained based on PC concatenation. To validate this approach, we simulated five hypothetical vertebral columns with serial variation based on rectangles (Additional file 1: Supplementary materials and methods). This analysis demonstrated that a morphospace based on Procrustes concatenation was better able to distinguish unique column morphologies than the traditional approach of plotting all vertebrae as independent elements (Additional file 1: Figures S3, S4), and that our approach produces identical results to concatenation of principal component scores (Additional file 1: Figures S4, S5, Table S4).

\section{Results}

\section{Whole-column shape}

The thoracolumbar column functions as an integrated unit, therefore it is important to consider variation in total thoracolumbar morphology. Combining Procrustes coordinates across five vertebral positions into a whole-column morphospace enables taxonomic and ecological groups to be distinguished (Fig. 3b and c; Additional file 1: Supplemental Materials and Methods). Landmarks from all the vertebral positions contribute to whole-column variation in the top three PCAs (those contributing $>5 \%$ variance, $51 \%$ total variance), highlighting the importance of extracting common patterns of variation along the column (Table 1).
Although Principal component axes from the whole-column PCA represent concurrent variation in all five vertebrae simultaneously, associated shape variation for each underlying vertebra was visualized by extracting the individual position loadings from the concatenated shape (Additional file 1: Figure S6). PC1 reflects variation from tall thoracics with large neural spines, and wide lumbars with elongate transverse processes (positive scores); to vertebrae with relatively smaller muscular processes throughout the column but larger metapophyses (negative scores) (Additional file 1: Figure S6). Positives scores on PC2 correspond to columns with wide-set (cervical-like) zygapophyses on T1, strongly anticlinal (varying from caudally to cranially directed) neural spines, and ventrally-inclined lumbar transverse process, typified by most euarchontoglires and some laurasiatheres (Additional file 1: Figure S6). Negative scores on PC2 represent columns with narrow-set (thoracic-like) zygapophyses on T1, caudally-directed neural spines throughout the column, and short, perpendicular lumbar transverse processes.

There are significant effects of phylogeny, size, and ecology on whole-column shape, corroborating Hypothesis 1 (Table 2). Although members of some clades cluster in the whole-column morphospace (Fig. $3 \mathrm{~b}$ ), the relatively low $\mathrm{K}$ value of 0.63 indicates deviation from pure Brownian motion (Table 2). Further, the distribution of taxa suggests considerable homoplasy, with certain clades (e.g., laurasiatheres) invading multiple regions of morphospace (Fig. 3a, b).

Post-hoc comparisons of ecological groups reveal significant differences in whole-column shape between terrestrial and fossorial, and to a lesser extent, scansorial-arboreal species $(p=0.028, p=0.056)$. Taxa utilizing fossorial or scansorial-arboreal habits tended to have low PC1 scores, reflecting relatively small to absent (in the case of monotremes) lumbar transverse processes and small neural processes, but extremely large metapophyses (Fig. 3, Additional
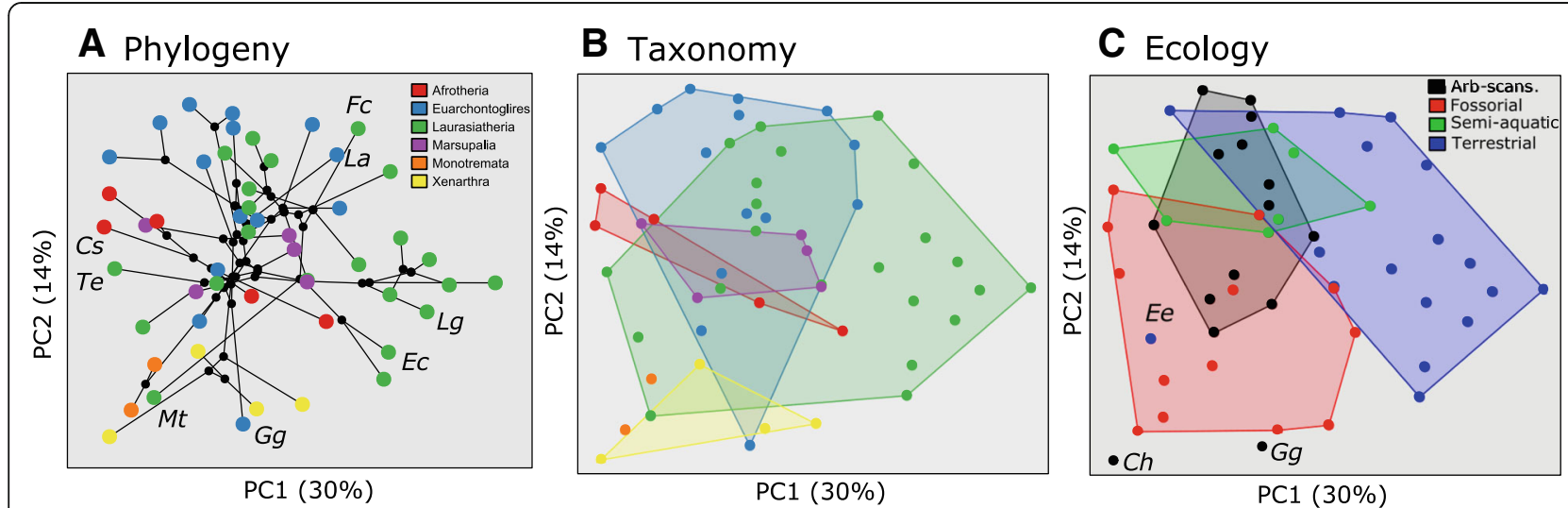

Fig. 3 Whole-column analysis of vertebral shape. PCA based on concatenated 'whole-column' shape (see Methods, Additional file 1: Supplementary Materials and Methods). A. Phylogenetic relationships plotted to create a phylomorphospace. Cs: Chyrsochloris stuhlmanni; Te: Talpa europaea; Mt: Manis temminckii; Gg: Gorilla gorilla; Ec: Equus caballus; Lg: Lama glama; La: Lepus americanus; Fc: Felis catus. B. grouped by Superorder; C. grouped by locomotor ecology. Ch: Choloepus hofmanni; Ee: Erinaceus europaeus; Gg: Gorilla gorilla 
Table 1 Summed loadings of landmarks from each vertebra on the whole-column PCA

\begin{tabular}{lllllll}
\hline & Tot Var. & T1 & Mid-T & Dia & Ant L & Last L \\
\hline PC1 & $30.0 \%$ & $22.0 \%$ & $23.2 \%$ & $16.1 \%$ & $20.6 \%$ & $18.2 \%$ \\
PC2 & $13.5 \%$ & $17.2 \%$ & $9.6 \%$ & $17.2 \%$ & $29.6 \%$ & $26.4 \%$ \\
PC3 & $7.5 \%$ & $25.9 \%$ & $23.5 \%$ & $20.2 \%$ & $16.3 \%$ & $14.1 \%$
\end{tabular}

Tot var. Total variation explained by that PC, T1 First thoracic, Mid-T midthoracic, Dia diaphragmatic, Ant $L$ anterior lumbar, Last $L$ last lumbar

file 1: Figure S6, S7). Terrestrial taxa have high PC1 scores indicating larger neural spines and transverse processes. Repeated invasion of low $\mathrm{PC} 1$ regions of morphospace indicates convergent evolution of this morphology several times within Mammalia in association with digging or climbing ecologies. For example, the highly fossorial taxa Talpa europaea and Chrysochloris stuhlmanni, (Fig. 3a: Te, Cs) lie on the far negative PC1, despite being members of distantly-related clades. Similarly, the pangolin Manis temminckii (Fig. 3a: Mt) lies at negative PC1 near other fossorial taxa (e.g., monotremes), and far from its sister group the Carnivora.

Among the terrestrial taxa, PC2 appears to distinguish dorsostable ungulates (e.g., Equus caballus, Llama glama; Fig. 3a: Ec, Lg) from dorsomobile runners (e.g., Felis catus, Lepus americanus; Fig. 3a: Fc, La). This variation reflects elongate and obliquely oriented transverse processes and cranially-inclined neural spines in the dorsomobile species (high PC2), and long but perpendicularly oriented transverse processes in the dorsostable species (low PC2) (Additional file 1: Figure S6).

Gorilla gorilla and Choloepus hoffmanni are outliers from the arboreal group, with relatively negative PC1 scores, likely relating to their suspensory mode of locomotion (Fig. 3c: Ch, Gg). Similarly, the hedgehog Erinaceus europaeus is also an outlier from the terrestrial group, likely reflecting phylogenetic inertia as it clustered with other ecologically-disparate eulipotyphlans (Fig. 3c: Ee), instead of species with more similar ecologies.

\section{Individual vertebral shape}

A more complex story is revealed when evolutionary factors are broken down by vertebral position. Although phylogeny and size have a significant effect at all five vertebral positions, ecology has a significant effect only at the last two positions (anterior lumbar and last lumbar) (Table 2, Fig. 4). This finding corroborates Hypothesis 2 in suggesting enhanced ecological influences in the posterior column. Post-hoc pairwise tests indicate significant differences between terrestrial and scansorial-arboreal-fossorial taxa in the lumbar region (Anterior: $\mathrm{P}_{\mathrm{Arb}-\mathrm{Ter}}=0.030, \mathrm{P}_{\mathrm{Fos}-}$ s-Terr=0.033; Last: $\mathrm{P}_{\text {Arb-Terr }}=0.034, \mathrm{P}_{\text {Foss-Terr }=0.024)}$ are driving this pattern. In PCA space, the terrestrial group (blue) has more negative PC1 scores than scansorial-arboreal (red) and fossorial (black) groups at the last two vertebral positions (Fig. 4). Terrestrial taxa (negative PC1) are typified by longer neural spines and transverse processes, which are cranio-ventrally deflected; whereas arboreal and fossorial taxa are characterized by short processes and large metapophyses (Additional file 1: Figure S7).

\section{Modularity}

Trunk vertebrae are significantly modular, both with $(\mathrm{CR}=0.798, p=0.001)$ and without $(\mathrm{CR}=0.748, p=$ 0.001) phylogenetic correction, indicating that despite strong covariation along the column vertebrae can still evolve somewhat independently. The $\mathrm{CR}$ ratios are below one, suggesting relative independence between vertebral positions. While between-vertebra modularity based on raw data reflects a simple relationship with vertebral position (closer vertebrae more integrated), phylogenetic modularity reflects are more complex pattern (Fig. 5a). The first three positions (T1 to diaphragmatic) and last two positions (anterior and last lumbar) have higher $\mathrm{CR}$ values, and thus are less independent than any other combinations. This supports the prediction that thoracic vertebrae and lumbar vertebrae form semi-independent modules (Hypothesis 3).

Table 2 Effects of phylogeny, size, and ecology on whole-column morphology and individual vertebral positions

\begin{tabular}{|c|c|c|c|c|c|c|c|c|}
\hline \multirow[t]{2}{*}{ Vertebral Position } & \multicolumn{2}{|c|}{ Phylogeny } & \multicolumn{2}{|c|}{ Allometry } & \multicolumn{2}{|c|}{ Ecology } & \multicolumn{2}{|c|}{ Ecology 2} \\
\hline & $\mathrm{K}$ & $p$-val. & Rsq & $p$-val. & Rsq & $p$-val. & Rsq & $p$-val. \\
\hline Whole Column & 0.63 & 0.001 & 0.12 & $<0.001$ & 0.09 & 0.012 & 0.08 & 0.032 \\
\hline First Thoracic & 0.65 & 0.001 & 0.16 & $<0.001$ & 0.07 & 0.112 & 0.07 & 0.174 \\
\hline Mid Thoracic & 0.72 & 0.001 & 0.14 & $<0.001$ & 0.08 & 0.086 & 0.08 & 0.066 \\
\hline Diaphragmatic & 0.54 & 0.001 & 0.10 & $<0.001$ & 0.05 & 0.535 & 0.06 & 0.331 \\
\hline Second Lumbar & 0.63 & 0.001 & 0.13 & $<0.001$ & 0.11 & 0.006 & 0.08 & 0.051 \\
\hline Final Lumbar & 0.66 & 0.001 & 0.10 & $<0.001$ & 0.13 & 0.001 & 0.09 & 0.048 \\
\hline
\end{tabular}

Phylogenetic signal was measured using the K-statistic. Allometry, ecology, and their interaction from a phylogenetic MANCOVA. Rs $q$ R-squared value. P-val. $p$ value, Ecology2 secondary ecology assignment used

Bold values indicate significance at the 0.05 alpha level 

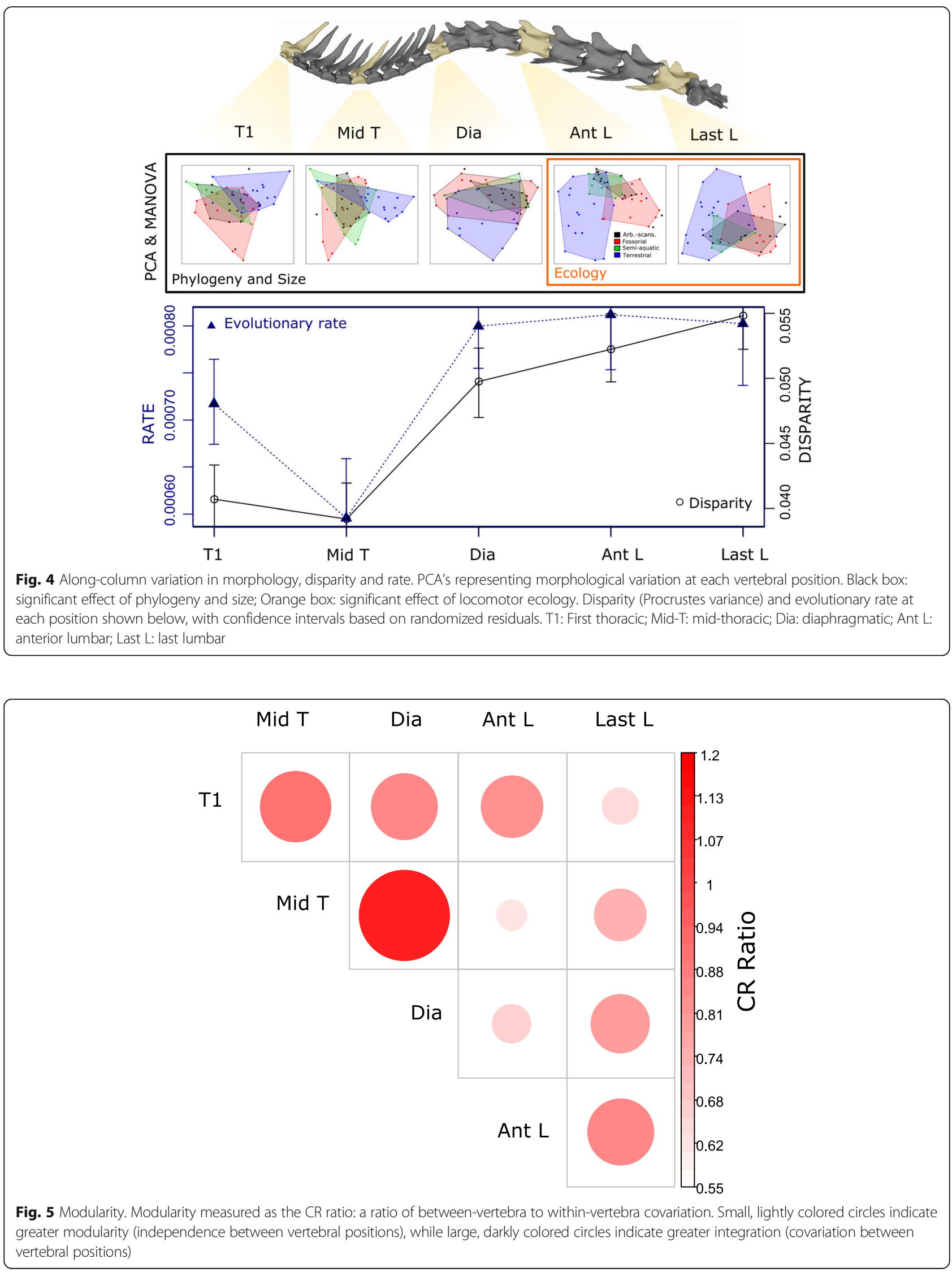


\section{Disparity and evolutionary rate}

Disparity and evolutionary rate both increase posteriorly along the thoracolumbar column. Pairwise comparisons reveal that the lumbar positions have significantly higher Procrustes variance than the first two thoracic positions (Table 3, Fig. 4), corroborating Hypothesis 4. The diaphragmatic vertebra exhibits intermediate levels of disparity. Similarly, rates of evolution are also greatest in the posterior trunk, with the last three positions exhibiting significantly higher rates than the first or mid- thoracic positions (Table 3, Fig. 4).

\section{Discussion}

Using geometric morphometrics, we quantified morphological variation in serially-homologous vertebrae to examine how vertebral regionalization has impacted adaptation and evolvability of the mammalian thoracolumbar column. Although the effects of phylogeny and size appear to be consistently important throughout the mammalian trunk, the evolutionary response of vertebral morphology to ecology is partitioned between vertebral positions, implicating vertebral regionalization as an important mechanism facilitating adaptation of the vertebral column to divergent ecological and locomotor functions.

\section{Phylogeny and size constraints}

Similarity due to shared common ancestry ('phylogeny') is a major factor driving the patterns of morphological variation among species. The results presented here support previous work demonstrating the importance of phylogeny to vertebral variation $[25,27,31]$. Recovered K-values ranged between 0.55 and 0.65 , indicating that although the effect of phylogeny was significant in shaping mammalian vertebral morphology, the variation in structure differed from that expected under the null hypothesis of Brownian motion [58, 59]. Variation in size places structural constraints on the axial skeleton due to its role in resisting gravitational loads [28, 30, 71], resulting in allometry in axial structure and function within groups (e.g., felids [31, 72, 73], bovids [25, 32], and

Table 3 Pairwise $p$-values for between-position differences in disparity (upper) and evolutionary rate (lower)

\begin{tabular}{lllllll}
\hline & & \multicolumn{3}{l}{ Disparity } & & \\
\cline { 3 - 7 } & & T1 & Mid-T & Dia & Ant L & Last L \\
\hline Evo. Rate & T1 & 1.000 & 0.768 & 0.084 & $\mathbf{0 . 0 2 9}$ & $\mathbf{0 . 0 0 8}$ \\
& Mid-T & $\mathbf{0 . 0 0 1}$ & 1.000 & 0.051 & $\mathbf{0 . 0 1 4}$ & $\mathbf{0 . 0 0 2}$ \\
& Dia & $\mathbf{0 . 0 2 5}$ & $\mathbf{0 . 0 0 1}$ & 1.000 & 0.638 & 0.327 \\
& Ant L & $\mathbf{0 . 0 1 2}$ & $\mathbf{0 . 0 0 1}$ & 0.761 & 1.000 & 0.621 \\
& Last L & $\mathbf{0 . 0 2 2}$ & $\mathbf{0 . 0 0 1}$ & 0.931 & 0.807 & 1.000 \\
\hline
\end{tabular}

$T 1$ First thoracic, Mid-T mid-thoracic, Dia diaphragmatic, Ant $L$ anterior lumbar, Last $L$ last lumbar

Bold values indicate significance at the 0.05 alpha level kangaroos [27]). We further confirm this finding on a broader evolutionary scale by showing a significant influence of size on both the whole-column and individual vertebral positions in our cross-mammalian sample (Table 2).

\section{Ecological effects}

Mammals employ diverse locomotor strategies to exploit their environment, which is often reflected in their postcranial anatomy [52, 65, 74-78]. Our results indicate a significant effect of locomotor ecology in the thoracolumbar column, even after phylogenetic and size correction (Table 2). The importance of the axial system in locomotion is highlighted by the major patterns of functional variation recovered in this analysis. The most striking trend in both the whole-column analysis (Fig. 3), and in the lumbar vertebrae (Fig. 4), was the contrast between terrestrial and fossorial/scansorial-arboreal morphologies. Interestingly, scansorial-arboreal and fossorial taxa partially overlapped in morphospace. This may reflect some overlap between these behaviors in certain taxa (e.g., Tamandua tetradactyla, Erithizon dorsatum, Manis temminckii), or similar functional demands placed on the axial skeleton by these seemingly disparate ecologies.

We hypothesize that the morphological patterns detected here reflect the relative importance of asymmetric (running) gaits in terrestrial taxa over symmetric (slower) gaits in fossorial/scansorial-arboreal species. Terrestrial taxa were typified by high PC1 scores in the whole column analysis, indicating elongated neural spines and transverse processes, whereas fossorial/scansorial-arboreal taxa tended to have lower PC1 scores consistent with well-developed metapophyses (Additional file 1: Figure S6). Enlarged metapophyses provide insertions for $\mathrm{mm}$. transversospinalis and deep portions of $M$. longissimus dorsi in therians [28], whereas $M$. longissimus dorsi is almost entirely absent in monotremes [79]. Therefore, the enlarged metapophyses in fossorial and some scansorial-arboreal groups may reflect enhanced stabilization of the trunk against strong limb motions during digging or climbing via $\mathrm{mm}$. transversospinalis (e.g., m. multifidus) and deeper insertions of M. longissimus [28], as well as restricted use of asymmetric gaits (which require an alternate arrangement of axial musculature) during non-terrestrial locomotion in these taxa.

Kinematic and anatomical data support this hypothesis. Comparative anatomical analyses of opossums, fossorial armadillos, and suspensory-arboreal sloths demonstrated significant increases in the size of $\mathrm{mm}$. transversospinalis and $m$. iliocostalis in the fossorial and arboreal species, with a relative reduction of $M$. longissimus [80, 81]. Likewise, kinematic data from the sloth (Choloepus didactylus) 
show both the absence of asymmetric gait-use, and reduction of sagittal bending in favor of lateral and torsional motion during arboreal locomotion relative to typical mammalian gaits [82]. Together, these data suggest that enlargement of $m$. transversospinalis and $m$. iliocostalis stabilizes the trunk against sagittal and lateral forces generated during digging and climbing behaviors, and that reduction of $M$. longissimus reflects the lack of asymmetric gaits that depend upon sagittal motions [80-82]. This suspensory-arboreal locomotor specialty is reflected in the divergent vertebral morphology of the sloth examined in this study, which lies at the minimum of both PC1 and PC2. Our data provide osteological evidence that the link between epaxial myology and behavior may be widespread among mammals, and bony features (e.g., enlarged metapophysis relative to neural spine and transverse processes) may be useful for identifying fossorial and arboreal behaviors in the fossil record.

Locomotor adaptation and evolvability in the lumbar region Although there was a significant influence of phylogeny and size throughout the trunk, variation associated with locomotor ecology was focused in the lumbar region (Table 2). The strong correlation of ecology and lumbar morphology reflects the important role of the lumbar region in mammalian locomotion. Whereas most tetrapods emphasize lateral bending of the trunk during locomotion, mammals employ sagittal bending during asymmetric gaits (e.g., galloping, bounding) or during leaping and jumping [50,83, 84]. This specialized mobility of the spine has been posited to be associated with the evolution of a ribless lumbar region because sagittal motions are restricted to the posterior trunk (though they are not perfectly correlated with the thoracolumbar transition), and the vertical orientation of the zygapophyses in this region is thought to facilitate sagittal bending $[83,85]$. In addition to the strong effect of ecology, there is also an increase in morphological disparity and evolutionary rates in the posterior trunk (Fig. 4). These results suggest natural selection for locomotor efficacy may be playing a role in driving rapid morphological evolution in the lumbar region, resulting in the acquisition of new, diverse morphologies.

A link between lumbar shape, locomotor ecology, and disparity has been proposed at a smaller phylogenetic scale within Felidae (cats) [31, 45]. However, unlike the felid dataset, which indicated significant ecological signal in the lumbar and diaphragmatic (transitional) regions, we did not find a significant correlation at the diaphragmatic position (Table 2, Fig. 4). Therefore, we hypothesize that the posterior thoracic vertebrae may be variably recruited into locomotor function with the lumbar region between taxa. This idea is supported by kinematic data showing that the cranial extent of sagittal bending during running varies, including posterior thoracics in some species but restricted to the lumbar region in others [83]. Due to their highly dorsomobile gait, felids likely involve the diaphragmatic region during running, resulting in the correlation between locomotor ecology and diaphragmatic morphology [86, 87].

\section{Gradational selection and modularity in the mammal trunk}

Relative to most other tetrapods, the mammalian vertebral column is strongly differentiated into regions with distinct morphologies. Two essential components are required to explain this heterogeneity: disparate selective regimes and modularity. Disparate selective regimes may be generated by selection gradients formed along the vertebral column. Due to its anatomical connection between the forelimb and hind limb, and its elongate structure, biomechanical forces in the trunk (and the vertebral column in particular) act in a strongly gradational manner. For example, mediolateral forces generated by the pelvis and hind limb during locomotion form a decreasing force gradient - from high caudally to low cranially [88]. Likewise, the ventral 'sagging' forces placed upon the trunk due to support of body mass between the limbs gradually reach a peak at the mid-trunk $[28,89]$, and sagittal bending contributing to pelvic displacement during mammalian asymmetric gaits increases posteriorly along the trunk [83]. These forces may generate highly heterogenous selection regimes for vertebrae at different positions, such that the selection pressures acting upon each vertebra may form a gradient (Fig. 6a, arrows), providing impetus for divergent evolution along the column.

Serially-homologous structures tend to covary strongly due to shared developmental origins, which can act as a developmental constraint on intracolumn variation [14, 90]. However, modularity, and the subdivision of the column into regions, provides a mechanism for limiting or modulating this constraint. In Mus, anteroposterior expression of Hox genes correlate with craniocaudal region boundaries in adult morphology, implicating these genes in controlling vertebral regionalization [91, 92]. Further, knock-out experiments suggest that Hox10 is crucial for patterning the ribless lumbar region in mammals [93]. This underlying developmental modularity is reflected by the evolutionary phenotypic modularity across the broad range of mammal taxa examined here, (Fig. 5, [23]), providing a mechanism for partitioned evolutionary responses to gradational selection (Fig. 6a).

Compared to mammals, the extinct forerunners of mammals, the non-mammalian synapsids, exhibit relatively homogeneous vertebral columns with little regional differentiation $[39,44,94]$. This suggests stronger integration between vertebrae (Fig. 6b), more uniform 


\section{Heterogenous}

A

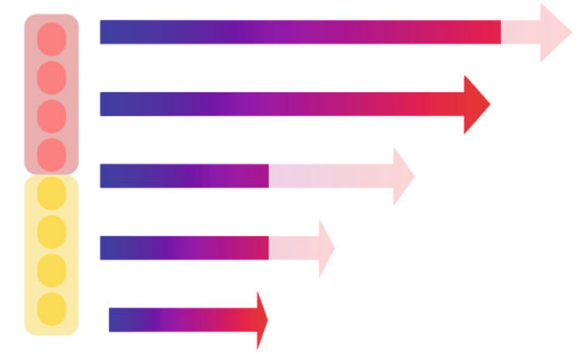

Homogenous

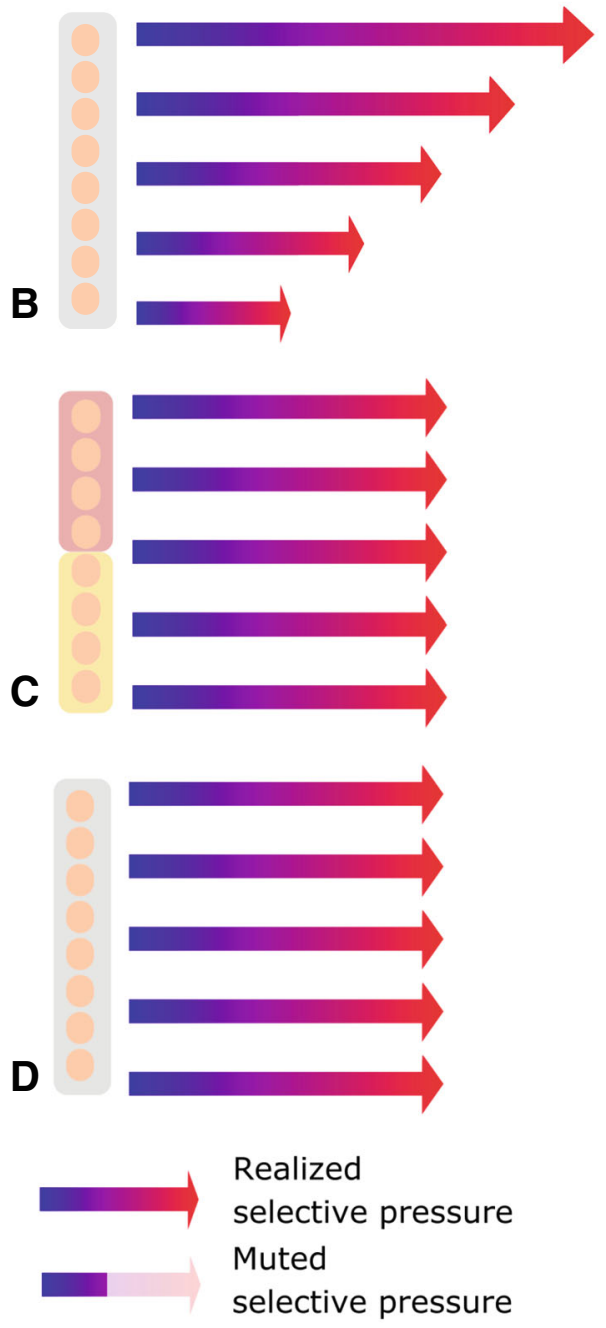

Homogenous

vertebrae

Heterogenous

vertebrae
Integration

Modularity
Fig. 6 Hypothesized relationship of selection and modularity in the axial skeleton. The strength of selection may vary along the vertebral column (length of arrows, a, c), but the evolutionary response to that selection is modulated by integration patterns (shading on arrows). Four different evolutionary scenarios are hypothesized. a Modularity and gradational selection. Selection varies along the column and the selective regimes of different modules may diverge (stronger in the red zone than the yellow zone) due to limited integration between red and yellow modules. Such a scenario is proposed to explain the heterogenous vertebral column of mammals. Homogeneous vertebral columns may result from either increased integration, uniform selection or both. $\mathbf{b}$ Integration and gradational selection. Selection regimes vary along the column, but morphological variation is muted by strong integration between vertebrae. c Modularity with uniform selection. Although the potential for generating evolutionary variation between vertebrae exists, selection maintains uniformity along the column. d Integration with uniform selection. Both factors limit craniocaudal variation. Circles: vertebrae; shaded boxes (yellow, red, grey): modules; colored arrows: selective pressure; greyed-out arrows: selective pressure muted by serial integration

selection (Fig. 6c), or a combination of these factors (Fig. $6 \mathrm{~d})$. Recent analyses of subtle gradients in vertebral morphology suggest that basal synapsids had fewer vertebral regions than crown therians, indicating that they may also have been less modular [44]. The appearance of the ribless lumbar region in Mesozoic mammals has been used to infer shifts in Hox function that could signal increasing vertebral modularity in mammalian evolution [95]. Although the evolution of vertebral function in fossil synapsids is poorly understood, basal members of the group likely lacked asymmetric gaits and sagittal vertebral flexion $[39,96]$. Therefore, therian mammals are derived in terms of both modularity and function, and either or both factors may play a role in the evolution of vertebral heterogeneity. Modularity itself may arise either by 'variational adaptation' - in which the evolution of reduced covariation between regions is a direct response to divergent selective regimes - or from the indirect erosion of developmental-genetic interactions between regions in response to selection for phenotypic robustness against pleiotropy [14]. Further work examining vertebral patterning and the relationship between regionalization and vertebral function in synapsids is required to tease apart this complex issue.

The data presented here suggest that locomotor selection for sagittal bending acting on the posterior trunk in mammals exploits modular variation to enable divergent evolution of vertebral regions. Modularity may lead to increased population-level and macro- evolutionary rates by aligning the genetic variation with the direction of selection, and limiting the interference between regions adapted for different functions $[4,15]$. Thus, the serial morphology of the mammalian vertebral column reflects both the gradational selection and the integration patterns imposed by its modular structure. 


\section{Conclusions}

Relative to other tetrapods, mammals have highly differentiated thoracic and lumbar trunk regions. Here we demonstrate that modularity of the thoracolumbar column in mammals is accompanied by divergence of evolutionary responses in serially homologous vertebrae. Overall, vertebral shape is influenced by phylogeny, size and ecological specialization. However, the important role of the mammalian lumbar region in locomotion is reflected in enhanced ecological adaptation. More significantly, our data show that elevated adaptability of the posterior trunk across mammals is linked with increased disparity and evolutionary rates. For example, evolution of fossorial and arboreal behaviors across the mammal tree resulted in repeated convergence of lumbar morphologies linked with the loss of asymmetric gaits. Strong gradational selection for behaviors such as these, coupled with modular variation, likely facilitated rapid and disparate evolution of the lumbar region in mammals.

\section{Additional file}

Additional file 1: Supplementary Materials and Methods, Figures S1-S7, Tables S1-S5, Supplementary references. (PDF 753 kb)

\section{Abbreviations \\ AMNH: American Museum of Natural History; CT: Computed tomography; FMNH: Field Museum of Natural History; GMM: Geometric morphometrics; GPA: Generalized Procrustes superimposition; MANCOVA: Multivariate analysis of covariance; MCZ: Museum of Comparative Zoology; PCA: Principal components analysis; T1: First thoracic}

\section{Acknowledgments}

We would like to thank Judy Chupasko and Mark Omura for help accessing the MCZ specimens used in this study. For access to specimens from online repositories we thank Tim Rowe, Jessica Maisano, Thomas Macrini, Jenifer Olori, Eric Ekdale (DigiMorph.org) and Doug Boyer (MorphoSource). We are grateful to Dave Sheets, David Polly, and Blake Dickson for advice regarding the wholecolumn analysis and thank two anonymous reviewers for their feedback on a draft of the manuscript. We thank Dean Adams for technical assistance with phylo.modularity.

\section{Funding}

Funding was provided by NSF grants EAR-1524523 (to S.E.P.) and EAR-1524938 (to K.D.A.), the American Association of Anatomists Postdoctoral Fellowship (K.E.J), and the Museum of Comparative Zoology Grants-in-aid of Undergraduate Research and Harvard College Research Program (L.B). Additional specimens were obtained from online repositories with funding as follows: Digimorph: NSF grants DEB-0309369 and IIS-0208675; Morphosource: NSF BCS 1317525 (to DM Boyer and ER Seiffert), 1552848 (to DM Boyer), and Duke University Trinity College of Arts and Sciences. These funding bodies played no role in study design, data collection, analysis, interpretation or manuscript preparation.

\section{Availability of data and materials}

The dataset supporting the conclusions of this article is available in the Dryad repository (https://doi.org/10.5061/dryad.g333421).

\section{Authors' contributions}

Study design (KEJ, SEP), data collection (KEJ, LB), data analysis (KEJ, LB), manuscript preparation and editing (KEJ, LB, KDA, SEP). All authors read and approved the final manuscript.
Ethics approval and consent to participate

Not applicable

\section{Consent for publication}

Not applicable

\section{Competing interests}

The authors declare that they have no competing interests.

\section{Publisher's Note}

Springer Nature remains neutral with regard to jurisdictional claims in published maps and institutional affiliations.

\section{Author details}

${ }^{1}$ Museum of Comparative Zoology and Department of Organismic and Evolutionary Biology, Harvard University, 26 Oxford Street, Cambridge, MA 02138, USA. 'Integrative Research Center, Field Museum of Natural History, 1400 South Lake Shore Drive, Chicago, IL 60605-2496, USA.

Received: 31 May 2018 Accepted: 30 October 2018

Published online: 16 November 2018

\section{References}

1. Atchley WR, Hall BK. A model for development and evolution of complex morphological structures. Biol Rev. 1991;66(2):101-57.

2. Bonner JT. The evolution of complexity by means of natural selection. Princeton: Princeton University Press; 1988.

3. Simon HA. The architecture of complexity. In: Facets of systems science. Princeton: Springer; 1991. p. 457-76.

4. Wagner GP, Altenberg L. Complex adaptations and the evolution of Evolvability. Evolution. 1996:50:967-76.

5. Pavličev M, Cheverud JM. Constraints evolve: context dependency of gene effects allows evolution of pleiotropy. Annu Rev Ecol Evol Syst. 2015:46:413-34

6. Cheverud JM. Quantitative genetics and developmental constraints on evolution by selection. J Theor Biol. 1984;110(2):155-71.

7. Cheverud JM. Developmental integration and the evolution of pleiotropy. Am Zool. 1996;36(1):44-50.

8. Goswami A. Morphological integration in the carnivoran skull. Evolution. 2006;60(1):169-83.

9. Wagner GP. Homologues, natural kinds and the evolution of modularity. Am Zool. 1996;36(1):36-43.

10. Winther RG. Varieties of modules: kinds, levels, origins, and behaviors. J Exp Zool A Ecol Genet Physiol. 2001;291(2):116-29.

11. Klingenberg CP. Morphological integration and developmental modularity. Annu Rev Ecol Evol Syst. 2008;39:115-32.

12. Goswami A, Smaers JB, Soligo C, Polly PD. The macroevolutionary consequences of phenotypic integration: from development to deep time. Philos Trans R Soc Lond Ser B Biol Sci. 2014;369(1649)20130254:1-15.

13. Goswami A, Polly PD. The influence of modularity on cranial morphological disparity in Carnivora and Primates (Mammalia). PLoS One. 2010;5(3).

14. Wagner GP, Pavlicev M, Cheverud JM. The road to modularity. Nat Rev Genet. 2007:8(12):921.

15. Pavlicev M, Kenney-Hunt JP, Norgard EA, Roseman CC, Wolf JB, Cheverud $J M$. Genetic variation in pleiotropy: differential epistasis as a source of variation in the allometric relationship between long bone lengths and body weight. Evolution. 2008;62(1):199-213.

16. Hansen TF. Is modularity necessary for evolvability?: Remarks on the relationship between pleiotropy and evolvability. BioSyst. 2003;69(2-3):83-94.

17. Larouche $\mathrm{O}$, Zelditch ML, Cloutier R. Modularity promotes morphological divergence in ray-finned fishes. Sci Rep. 2018;8(1):7278.

18. Fleming A, Kishida MG, Kimmel CB, Keynes RJ. Building the backbone: the development and evolution of vertebral patterning. Development. 2015; 142(10):1733-44.

19. McShea DW. Evolutionary change in the morphological complexity of the mammalian vertebral column. Evolution. 1993;47(3):730-40.

20. Flower WH. An introduction to the osteology of the Mammalia. London: MacMillan; 1885.

21. Owen R. On the archetype and homologies of the vertebrate skeleton: author; 1848. 
22. Rawls A, Fisher RE. Development and functional anatomy of the spine. In: Kusumi K, Dunwoodie SL, editors. The genetics and development of scoliosis. New York: Springer; 2010. p. 21-46.

23. Randau M, Goswami A. Morphological modularity in the vertebral column of Felidae (Mammalia, Carnivora). BMC Evol Biol. 2017;17(1):133.

24. Buchholtz EA. Modular evolution of the cetacean vertebral column. Evol Dev. 2007;9(3):278-89.

25. Jones KE. Evolutionary allometry of lumbar shape in Felidae and Bovidae. Biol J Linn Soc Lond. 2015;116(3):721-40.

26. Arnold P, Forterre F, Lang J, Fischer MS. Morphological disparity, conservatism, and integration in the canine lower cervical spine: insights into mammalian neck function and regionalization. Mammalian BiologyZeitschrift für Säugetierkunde. 2016;81(2):153-62.

27. Chen XM, Milne N, O'Higgins P. Morphological variation of the thoracolumbar vertebrae in Macropodidae and its functional relevance. J Morphol. 2005;266(2):167-81.

28. Slijper EJ. Comparative biologic-anatomical investigations on the vertebral column and spinal musculature of mammals. Verh K Ned Akad Wet Afd Natuurkd Tweede Reeks. 1946;42(5):1-128.

29. Jones KE, Pierce SE. Axial allometry in a neutrally buoyant environment: effects of the terrestrial-aquatic transition on vertebral scaling. J Evol Biol. 2016.

30. Jones KE. Evolutionary allometry of the thoracolumbar centra in felids and bovids. J Morphol. 2015;276(7):818-31.

31. Randau M, Goswami A, Hutchinson JR, Cuff AR, Pierce SE. Cryptic complexity in felid vertebral evolution: shape differentiation and allometry of the axial skeleton. Zool J Linnean Soc. 2016;178(1):183-202.

32. Halpert AP, Jenkins FAJ, Franks H. Structure and scaling of the lumbar vertebrae in African bovids (Mammalia, Artiodactyla). J Zool. 1987;211:239-58.

33. Long JH, Pabst DA, Shepherd WR, McLellan WA. Locomotor design of dolphin vertebral columns: bending mechanics and morphology of Delphinus delphis. J Exp Biol. 1997;200(1):65-81.

34. Bramble DM, Carrier DR. Running and breathing in mammals. Science. 1983; 219(4582):251-6

35. Arnold P, Esteve-Altava B, Fischer MS. Musculoskeletal networks reveal topological disparity in mammalian neck evolution. BMC Evol Biol. 2017; 17(1):251.

36. Randau M, Goswami A. Unravelling intravertebral integration, modularity and disparity in Felidae (Mammalia). Evol Dev. 2017;19(2):85-95.

37. Narita Y, Kuratani S. Evolution of the vertebral formulae in mammals: a perspective on developmental constraints. J Exp Zool Part B Mol Dev Evol. 2005;304B(2):91-106

38. Müller J, Scheyer T, Head J, Barrett P, Werneburg I, Ericson P, Pol D, Sánchez-Villagra M. The evolution of vertebral numbers in recent and fossil amniotes: the roles of homeotic effects and somitogenesis. Proc Natl Acad Sci U S A. 2010;107:2118-23.

39. Kemp TS. The origin and evolution of mammals. Princeton: Oxford University Press; 2005.

40. Asher R, Lin K, Kardjilov N, Hautier L. Variability and constraint in the mammalian vertebral column. J Evol Biol. 2011;24(5):1080-90.

41. Buchholtz E, Yang J, Bailin H, Laves S, Drozd L. Localization of the diaphragm and axial patterning in mammals. J Vert Paleontol. 2011;31:79.

42. Crompton A, Jenkins FA Jr. Mammals from reptiles: a review of mammalian origins. Annu Rev Earth Planet Sci. 1973:1(1):131-55.

43. Jenkins FA. Cynodont postcranial anatomy and prototherian level of mammalian organization. Evolution. 1970;24(1):230-52

44. Jones KE, Angielczyk KD, Polly PD, Head JJ, Fernandez V, Lungmus J, Tulga $\mathrm{S}$, Pierce SE. Fossils reveal the complex evolutionary history of the mammalian regionalized spine. Science. 2018;361:1249-52.

45. Randau M, Cuff AR, Hutchinson JR, Pierce SE, Goswami A. Regional differentiation of felid vertebral column evolution: a study of 3D shape trajectories. Org Divers Evol. 2017;17(1):305-19.

46. Shapiro LJ, Simons CVM. Functional aspects of strepsirrhine lumbar vertebral bodies and spinous processes. J Hum Evol. 2002;42(6):753-83.

47. Williams SA. Placement of the diaphragmatic vertebra in catarrhines: implications for the evolution of dorsostability in hominoids and bipedalism in hominins. Amer J Phys Anthrop. 2012;148(1):111-22.

48. Johnson SE, Shapiro LJ. Positional behavior and vertebral morphology in atelines and cebines. Amer J Phys Anthrop. 1998;105(3):333-54.

49. Alvarez A, Ercoli MD, Prevosti FJ. Locomotion in some small to mediumsized mammals: a geometric morphometric analysis of the penultimate lumbar vertebra, pelvis and hindlimbs. Zoology. 2013;116(6):356-71.
50. Harty $\mathrm{TH}$ : The role of the vertebral column during jumping in quadrupedal mammals. PhD Thesis. Oregon State University; 2010.

51. Hedges SB, Dudley J, Kumar S. TimeTree: a public knowledge-base of divergence times among organisms. Bioinformatics. 2006;22(23):2971-2.

52. Samuels JX, Meachen JA, Sakai SA. Postcranial morphology and the locomotor habits of living and extinct carnivorans. J Morphol. 2013:274(2):121-46.

53. Zelditch M, Swiderski D, Sheets HD, Fink W. Geometric Morphometrics for biologists: a primer. Boston: Elsevier Academic Press; 2004.

54. Klingenberg CP. Novelty and "homology-free" morphometrics: what's in a name? Evol Biol. 2008;35(3):186-90.

55. Head JJ, Polly PD. Evolution of the snake body form reveals homoplasy in amniote Hox gene function. Nature. 2015;520(7545):86-9.

56. Adams DC, Otarola-Castillo E. Geomorph: an R package for the collection and analysis of geometric morphometric shape data. Methods Ecol Evol. 2013:4:393-9.

57. R: R development Core team. A language and environment for statistical computing; 2009

58. Adams DC. A generalized $\mathrm{K}$ statistic for estimating phylogenetic signal from shape and other high-dimensional multivariate data. Syst Biol. 2014;63(5): 685-97.

59. Blomberg SP, Garland T Jr, Ives AR. Testing for phylogenetic signal in comparative data: behavioral traits are more labile. Evolution. 2003;57(4):717-45

60. Adams DC. A method for assessing phylogenetic least squares models for shape and other high-dimensional multivariate data. Evolution. 2014;68(9): 2675-88.

61. Goodall C. Procrustes methods in the statistical analysis of shape. J R Stat Soc Ser B Methodol. 1991:285-339.

62. Adams DC. Evaluating modularity in morphometric data: challenges with the RV coefficient and a new test measure. Methods Ecol Evol. 2016;7(5): $565-72$

63. Goswami A, Polly PD. Methods for studying morphological integration and modularity. The Paleontological Society Papers. 2010 16:213-243.

64. Denton JS, Adams DC. A new phylogenetic test for comparing multiple high-dimensional evolutionary rates suggests interplay of evolutionary rates and modularity in lanternfishes (Myctophiformes; Myctophidae). Evolution. 2015:69(9):2425-40.

65. Fabre AC, Cornette R, Goswami A, Peigné S. Do constraints associated with the locomotor habitat drive the evolution of forelimb shape? A case study in musteloid carnivorans. J Anat. 2015;226(6):596-610.

66. Ekdale EG. Morphological variation among the inner ears of extinct and extant baleen whales (Cetacea: Mysticeti). J Morphol. 2016;277(12):1599-615.

67. Dumont M, Wall CE, Botton-Divet L, Goswami A, Peigné S, Fabre A-C. Do functional demands associated with locomotor habitat, diet, and activity pattern drive skull shape evolution in musteloid carnivorans? Biol J Linn Soc. 2016:117(4):858-78

68. Adams DC, Collyer ML. Permutation tests for phylogenetic comparative analyses of high-dimensional shape data: what you shuffle matters. Evolution. 2015;69(3):823-9.

69. Rohlf FJ. Geometric morphometrics and phylogeny. Morphology, shape and phylogeny; 2002. p. 175-93.

70. Adams DC. Methods for shape analysis of landmark data from articulated structures. Evol Ecol Res. 1999;1(8):959-70.

71. Biewener AA. Biomechanical consequences of scaling. J Exp Biol. 2005; 208(9):1665-76.

72. Cuff AR, Sparkes EL, Randau M, Pierce SE, Kitchener AC, Goswami A Hutchinson JR. The scaling of postcranial muscles in cats (Felidae) I: forelimb, cervical, and thoracic muscles. J Anat. 2016;229(1):128-41.

73. Cuff AR, Sparkes EL, Randau M, Pierce SE, Kitchener AC, Goswami A, Hutchinson JR. The scaling of postcranial muscles in cats (Felidae) II: hindlimb and lumbosacral muscles. J Anat. 2016:229(1):142-52.

74. White JL. Indicators of locomotor habits in xenarthrans: evidence for locomotor heterogeneity among fossil sloths. J Vert Paleontol. 1993;13(2): 230-42.

75. Gould FDH. The morphology of the distal femoral articular surface and the evolution of cursoriality in ungulates: PhD thesis. Johns Hopkins University; 2013.

76. Van Valkenburgh B. Skeletal indicators of locomotor behavior in living and extinct carnivores. J Vert Paleontol. 1987;7(2):162-82.

77. MacLeod N, Rose KD. Inferring locomotor behavior in Paleogene mammals via eigenshape analysis. Am J Sci. 1993;293(A):300.

78. Young JW, Danczak R, Russo GA, Fellmann CD. Limb bone morphology, bone strength, and cursoriality in lagomorphs. J Anat. 2014;225(4):403-18. 
79. Vallois HV. Les transformations de la musculature de l'épisome chez les vertébrés. Princeton: L'Universite d Paris; 1922.

80. Nyakatura JA, Stark H. Aberrant back muscle function correlates with intramuscular architecture of dorsovertebral muscles in two-toed sloths. Mammalian Biology-Zeitschrift für Säugetierkunde. 2015;80(2):114-21.

81. Gaudin TJ, Nyakatura JA. Epaxial musculature in armadillos, sloths, and opossums: functional significance and implications for the evolution of back muscles in the Xenarthra. J Mamm Evol. 2017:1-8.

82. Nyakatura JA, Fischer MS. Functional morphology and three-dimensional kinematics of the thoraco-lumbar region of the spine of the two-toed sloth. J Exp Biol. 2010;213(24):4278-90.

83. Schilling N, Hackert R. Sagittal spine movements of small therian mammals during asymmetrical gaits. J Exp Biol. 2006;209(19):3925-39.

84. Shapiro LJ. Functional morphology of the vertebral column in primates. In: Gebo DL, editor. Postcranial adaptation in nonhuman Primates. DeKalb: Northern Illinois University Press; 1993. p. 121-49.

85. Boszczyk BM, Boszczyk AA, Putz R. Comparative and functional anatomy of the mammalian lumbar spine. Anat Rec. 2001;264:157-68.

86. Macpherson JM, Ye Y. The cat vertebral column: stance configuration and range of motion. Exp Brain Res. 1998;119(3):324-32.

87. Gál JM. Mammalian spinal biomechanics 1: static and dynamic mechanicalproperties of intact intervertebral joints. J Exp Biol. 1993;174:247-80.

88. Schilling N, Carrier DR. Function of the epaxial muscles in walking, trotting and galloping dogs: implications for the evolution of epaxial muscle function in tetrapods. J Exp Biol. 2010;213(9):1490-502.

89. Smit TH. The use of a quadruped as an in vivo model for the study of the spine: biomechanical considerations. Eur Spine J. 2002;11(2):137-44.

90. Young NM, Hallgrimsson B. Serial homology and the evolution of mammalian limb covariation structure. Evolution. 2005;59(12):2691-704.

91. Wellik DM. Hox genes and vertebrate axial pattern. Curr Top Dev Biol. 2009; 88:257-78.

92. Wellik DM. Hox patterning of the vertebrate axial skeleton. Dev Dyn. 2007; 236(9):2454-63.

93. Wellik DM, Capecchi MR. Hox10 and Hox11 genes are required to globally pattern the mammalian skeleton. Science. 2003;301(5631):363-7.

94. Jenkins FA Jr. The postcranial skeleton of African Cynodonts. Bull Peabody Mus Nat Hist. 1971:36.

95. Luo ZX, Chen PJ, Li G, Chen M. A new eutriconodont mammal and evolutionary development in early mammals. Nature. 2007:446(7133):288-93.

96. Carrier DR. The evolution of locomotor stamina in tetrapods: circumventing a mechanical constraint. Paleobiology. 1987;13(3):326-41.

Ready to submit your research? Choose BMC and benefit from:

- fast, convenient online submission

- thorough peer review by experienced researchers in your field

- rapid publication on acceptance

- support for research data, including large and complex data types

- gold Open Access which fosters wider collaboration and increased citations

- maximum visibility for your research: over $100 \mathrm{M}$ website views per year

At $\mathrm{BMC}$, research is always in progress.

Learn more biomedcentral.com/submissions 